\title{
HIGH-ENERGY PHENOMENA IN BE STARS
}

\author{
(Review Paper)
}

Theodore P. Snow

Center for Astrophysics and Space Astronomy

University of Colorado, Boulder CO 80309

\section{INTRODUCTION}

It was discovered in the early years of the Copernicus satellite operations that the stellar winds in early-type stars are often characterized by degrees of ionization inconsistent with the spectral types of the stars. The most notable early example was the discovery of strong OVI lines in the form of $\mathrm{P}$ Cygni profiles in stars too cool to have this ion in their photospheres, such as $\tau$ Sco (BOV; Rogerson and Lamers 1975). Systematic studies showed that excess ionization is a general property of the winds in OB stars (e.g. Lamers and Snow 1978).

When Be stars began to be observed in the ultraviolet it was found that many of them have stellar winds, and furthermore that excess ionization was also characteristic of these winds, as it was in the more luminous $O$ and $B$ stars. This excess ionization, which today is often called "superionization", along with the associated stellar wind phenomena, forms the basis for this review. Here the term "high-energy phenomena" is taken to mean any phenomena requiring energies higher than can be derived from radiative equilibrium with the stellar photosphere. Hence this paper discusses superionization, stellar winds and all their characteristics, and high-energy (x-ray) emission. None of these phenomena would occur in radiative equilibrium with the stellar photosphere, and therefore they qualify as high-energy phenomena. One of the themes of this review will be the comparison between $\mathrm{Be}$ and $\mathrm{OB}$ stars in terms of high-energy phenomena.

\section{SUPERIONIZATION}

As already noted, "superionization" refers to the presence of ionization stages higher than can be formed in equilibrium at the temperature of the photosphere. In OB stars, the most widely observed such ions are OVI, which is observed to spectral types as late as B0; and NV, observed to spectral type B1 or B2. For stars near the B main sequence, CIV and SiIV are anomalously strong and indicate the presence of superionization. The IUE satellite has provided most of the available data, since Copernicus was relatively insensitive, especially at the wavelengths of CIV and SiIV. Nevertheless, the first recognition of winds and superionization in Be stars came from Copernicus observations (Lamers and Snow 1978; Snow and Marlborough 1976; Marlborough 1977).

There have now been several surveys of winds and superionization in $\mathrm{Be}$ stars, based on IUE data. These studies all show that Be stars stand out from normal $\mathrm{B}$ main sequence objects in having a greater degree of superionization (Marlborough and Peters 1982; Slettebak and Carpenter 1983; Barker, Marlborough, and Landstreet 1984; Grady, Bjorkman, and Snow 1987). These surveys show that CIV is seen in Be stars as 
late as B9, whereas this ion shows up in normal B stars only through type B2. Similarly, SiIV in Be stars extends to B9, but is weak or absent in normal B stars of this type.

The distinction between $B$ and $B e$ stars with respect to superionization is probably very significant, although the significance will best be appreciated when the cause of the superionization is known. Apparently whatever the cause is, it is somehow linked with the Be phenomenon itself. Among the OB stars, the prevalent theories for explaining superionization involve ionization by soft x-rays produced either in a corona (Cassinelli, Olson, and Stalio 1978; Cassinelli and Olson 1979; Cassinelli 1979) or by shocks in the wind (Lucy and White 1980; Lucy 1982). This raises the interesting question of possible similar x-ray emission from Be stars, which will be addressed in a later section.

There appears to be an intimate link between superionization and the presence of winds in Be stars. Winds are rarely, if ever, present without superionization, and it seems likely that superionization always arises only in the winds. The surveys of superionization in Be stars (cited above) typically use equivalent width measures to compare the CIV and SiIV strengths in Be stars with those in normal B stars, so this alone does not provide information on whether the excess ionization in Be stars arises in the winds. But examination of the profiles of the CIV and SiIV lines usually shows asymmetries or shifted discrete absorption components having substantial outflow velocities, suggesting that most or all of the ion arises in circumstellar material that is expanding away from the stars. There are several observational difficulties in evaluating these line profiles, however, which are discussed in the next section.

\section{WINDS IN Be STARS}

The first recognition that Be stars have high-velocity winds came about as part of the mid-1970s surveys of OB-star mass loss in general, based primarily on Copernicus data (e.g. Snow and Marlborough 1976; Morton 1976; Snow and Morton 1976). At first it was not known whether Be stars stood out from normal B stars, but this became evident as efforts were made to explore the lower range of luminosities where winds occur (e.g. Snow and Marlborough 1976; Marlborough 1977; Lamers and Snow 1978). Now the large-scale surveys based on IUE data have made it abundantly clear that winds are stronger in Be stars than in normal B stars of similar type (Marlborough 1982; Slettebak and Carpenter 1983; Barker, Marlborough, and Landstreet 1984; Grady, Bjorkman, and Snow 1987). Normal B stars, at least among the earlier types (B2 and earlier) can have winds, but where they do, they appear to be weaker than in Be stars of similar type.

Two types of line profiles indicate the presence of winds in Be stars: (1) asymmetric, broad absorption in resonance lines; and (2) shifted discrete absorption components, most commonly seen in CIV and SiIV. It is claimed by some (e.g. Henrichs 1986; Grady, Bjorkman, and Snow 1987) that in fact all of the wind absorption consists of discrete components, so that the broad asymmetric profiles are composites of a number of such components. Examination of some of the early Copernicus line profiles (e.g. Snow and Marlborough 1976) casts some doubt on this conclusion, because structure in the extended short-wavelength wings should have been seen if in fact the profiles consisted entirely of discrete components. This point will be discussed further, in the section on discrete absorption components. 
a. Broad Asymmetric Absorption Profiles

The general view of the asymmetric resonance line profiles with extended short-wavelength wings is that they represent the extension to low mass-loss rates of the classical P Cygni profiles that occur in very luminous OB stars. Observations and theory show that, for low mass-loss rates, the emission part of the profile is diminished and the optical depth in the short-wavelength wing is decreased, producing only an asymmetric absorption profile (theoretical profiles may be computed following Castor and Lamers 1979, for example).

It has been recognized for some time (cf. remarks in Snow 1981) that serious ambiguities are introduced into the interpretation of the extended short-wavelength wings by observational difficulties. As the optical depth in the wind decreases with distance from line center, the profile gradually merges into the continuum, and it is sometimes difficult or impossible to say with certainty where the edge of the profile is. This makes the determination of terminal velocities difficult, for example, and can also affect the derivation of mass-loss rates.

Recently an even more extreme warning has been sounded by Hubený, Harmanec, and Stefl (1986), who argue that line blending can simulate line asymmetries that are actually completely absent. They make a strong case for this point of view by calculating theoretical spectra and comparing them to $I U E$ observations that have been interpreted by others in terms of stellar winds, and showing that the observed spectra are consistent with the calculated ones, which include no wind absorption. While the point made by Hubený et al. is well taken, it is clear that asymmetric broad resonance lines do exist in Be stars, because (1) the calculated profiles of Hubený et al. do not always fit well enough; (2) they ignore variability; and (3) they used only IUE data, which are too noisy to allow stringent profile-fitting analysis. Higher-resolution, higher-quality Copernicus data (such as shown by Snow and Marlborough 1976) show that the broad absorption features are not blends of narrow lines. Nevertheless, this issue should be kept in mind whenever winds are weakly or marginally indicated.

The ions most commonly seen to form broad asymmetric absorption lines are CIV $(\lambda \lambda 1548,1550)$ and SiIV $(\lambda \lambda 1394,1403)$, which are present throughout the Be stars to B9. For the hotter Be stars, NV $(\lambda \lambda 1238,1242)$ or even OVI $(\lambda \lambda 1032$, 1038) may appear, while for mid- to late-B stars, SiIII ( $\lambda$ 1206) may have an extended short-wavelength wing. The latter ion is quite significant, because the presence of SilII simultaneously with SiIV provides the best information available on the degree of ionization in the winds. Unfortunately, SiIII is not well observed with IUE, and was seen only in a few cases with Copernicus (Snow 1981). Furthermore, the SillI line is even more prone to confusion with blends and problems with locating the continuum than are CIV and SiIV, so at present the information based on SillI is not very reliable. Detailed study of this and the other resonance lines in Be stars will, no doubt, be a profitable task with the Hubble Space Telescope at its highest resolution.

\section{b. Mass-Loss Rates}

Several studies of mass-loss rates from Be stars have been based on analyses of ultraviolet resonance lines (e.g. Marlborough 1982; Snow and Stalio 1987 and references cited therein). The technique used is to fit calculated line profiles to observed ones, varying such parameters as the optical depth in the wind, the velocity law, and the rate of change 
of ionization with height. Theoretical profiles can be calculated from the very useful paper of Castor and Lamers (1979). When the winds are optically thin, as is the case for most Be stars, the principal uncertainties in the calculation of mass-loss rates are (1) the terminal velocity, which is difficult to determine because of the continuum problem discussed above; (2) the ionization balance, although this is not so bad if both SiIII and SiIV are seen; (3) the geometry of the wind, which is usually assumed spherically symmetric but which may very well be axisymmetric or even spotty; and (4) the omission of discrete wind features from the calculation.

Wind terminal velocities as high as $1000 \mathrm{~km} \mathrm{~s}^{-1}$ (or slightly higher) have been observed in Be stars, whereas values as high as 2000 to $3000 \mathrm{~km} \mathrm{~s}^{-1}$ are found in the more luminous $O B$ stars. Again, it is stressed that the actual values for many Be stars are probably higher than can be determined from the edge of the absorption profile, because the wind becomes too optically thin to be observed beyond a certain velocity.

Typical values for the mass-loss rates derived for Be stars from ultraviolet lines lie in the range $10^{-11}$ to $10^{-9}$ solar masses per year (e.g. Snow 1981; many references in Jaschek and Groth 1982; references cited in Snow and Stalio 1987). As pointed out by Snow (1982), these values are roughly consistent with what would be expected from the mass-loss rates for the more luminous $O B$ stars; that is, if the empirical relationship between mass-loss rate and luminosity is extended to the lower luminosities characteristic of Be stars, the Be stars lie fairly near (but usually below) the relation. This suggests that radiation pressure may play a similar role in Be star winds to that in OB star winds, but the scatter among Be stars argues for the existence of other factors that at least modify the mass loss.

Attempts to find correlations between mass loss and other parameters in Be stars generally fail (e.g. Snow 1981). One of the parameters scrutinized most extensively is the line-broadening measure vsini, but plots of mass-loss rate versus vsini are generally scatter diagrams. This may be interpreted as due to a lack of latitude-dependence, if $\mathrm{Be}$ stars are all rapid rotators; or a lack of dependence on rotational velocity, if $\mathrm{Be}$ stars have a wide range of rotations. Correlation studies are further complicated by time variability, which certainly creates scatter in any correlation plot, and by radiative transfer effects in the atmospheres of rotating stars, which confuse the interpretation of vsini. As discussed in the next section, some meaningful information is derived from correlation studies involving the discrete absorption components that often characterize Be star winds.

The mass-loss rates found from ultraviolet resonance lines are often far smaller than those derived from infrared continuum flux data. This was pointed out by several authors at the last Be star symposium (Jaschek and Groth 1982), and is discussed at this colloquium as well, mostly by Lamers and by Waters. The probable reason for the disparity is that the infrared excess emission arises in a physically different region than the ultraviolet wind absorption features. Notably, this explanation works only if the infrared region or the ultraviolet region (or both) is not spherically symmetric, or if mass flux is not constant throughout both regions.

The nonspherical explanation is favored by most workers, who adopt a disk model in which the infrared emission arises in an equatorially-concentrated circumstellar zone of high density, and the ultraviolet wind features are formed over a more extended region outside the plane of the disk (these models are stimulated largely by the presence 
of optical shell absorption lines in some stars and by polarization measurements). There is, however, at least one spherically symmetric model that explains the mass-loss rate discrepancy as due to time variable behavior; the suggestion is that mass builds up over long periods of time in the infrared-emitting region (which is outside of the high-velocity wind), occasionally dispersing into the surrounding medium (Doazan and Thomas, this conference, and references cited therein). The disk models have the advantage that they account for the polarization, while the time-variable model has the advantage that it incorporates the episodic behavior observed in many Be stars on timescales of decades. It is worth reemphasizing that the differences in mass-loss rates derived by the two methods almost certainly show that one of these two classes of models is required.

\section{c. Discrete Absorption Components}

Relatively narrow (FWHM $\sim 10^{2} \mathrm{~km} \mathrm{~s}^{-1}$ ) absorption components were observed superposed on the broad P Cygni features in many OB stars in the early Copernicus surveys (e.g. Snow and Morton 1976). These features have been examined in detail by Lamers, Gathier, and Snow (1982) and more recently by Prinja and Howarth (1986), with the general result that these components may be present in all OB stars, that they tend to appear at about the same velocity at all times in a given star, that they represent only a small fraction of the mass loss, that they are variable in strength, and that the degree of ionization in the material that forms the components is higher than in the steady wind represented by the broad P Cygni features.

The presence of discrete absorption components in Be stars was recognized as soon as $I U E$ data became available. It was also quickly recognized that time variability, not just in strength but also in wavelength, is a common feature of these components in Be stars. In this section some characteristics of the discrete components are discussed, but much of their behavior will be described in the next section, on time variability in general.

The ions in which the discrete components are most commonly observed are CIV and SiIV, although they can be seen in NV in the hottest Be stars. The velocities of the components range from near the rest velocity of the star to roughly $1000 \mathrm{~km} \mathrm{~s}^{-1}$, and at the same time a given star may exhibit multiple components at different velocities.

As noted earlier, it has been argued that the broad asymmetric resonance lines in Be stars consist entirely of blended discrete components (Henrichs 1986; Grady, Snow, and Bjorkman 1987), but this conclusion should be modified. It may be correct to say that all of the excess CIV and SiIV absorption in Be stars (compared with normal B stars) is due to discrete components.

Studies of discrete absorption components in Be stars have tended to fall into two categories: (1) detailed studies of individual stars, which always involve time variability and will therefore be discussed in the next section; and (2) surveys of large numbers of stars. The most recent example of the latter, by Grady, Bjorkman, and Snow (1987) incorporated $I U E$ data on $62 \mathrm{Be}$ and 45 normal B stars.

The survey by Grady et al. and an earlier study by Henrichs (1984) showed that discrete absorption components among B stars are phenomena reserved for Be stars exclusively. Normal B stars do not show these components, whereas they were found in at least one spectrum of roughly two-thirds of the Be stars (and may appear in all Be stars in time). Thus it seems that the presence of discrete absorption components is a very important key to the Be phenomenon. 
The discrete absorption components show a correlation with vsini, in the sense that there is a threshold, at vsini $\sim 150 \mathrm{~km} \mathrm{~s}^{-1}$, below which the components are not seen. This argues for a degree of equatorial confinement of the features, if Be stars are all intrinsically rapid rotators; or for a threshold rotational velocity for formation of the features. At the same time shell stars, which are usually presumed to be seen nearly equator-on, show little or no evidence for high-velocity winds; if discrete components are present, they lie at low velocities (Grady et al.). This combination of findings implies that there is indeed a disk in which high-velocity mass flow does not occur, and that the winds also do not reach very high latitudes.

\section{TIME VARIABILITY}

It is a well-known fact that $B e$ stars are variable on many timescales, and the high-energy phenomena discussed in this review are no exception. Because ultraviolet observations are required to observe these phenomena, however, less is known about their variability than the variability of optical features, particularly on timescales of decades. The available data base is getting long enough, though, that some information is now in hand on long-term behavior.

It was noted in some of the early Copernicus studies that the resonance lines in Be stars are prone to variation, although little was known about the timescales. Typically a given star would be reobserved, if at all, months or even years after its initial observation. In one case (59 Cygni) Marlborough and Snow (1980) happened to discover significant changes in the NV profile in less than an hour, leading to the suspicion that the changes seen over long times were actually due to short-term variations. Some attempts were made to seek short-term variability (e.g. Slettebak and Snow 1978), but little was seen, indicating that rapid variability was not a full-time phenomenon.

Again, IUE observations have provided much broader coverage than earlier instruments, and in the nine years of $I U E$ operations some progress has been made in understanding the nature and the timescales of the variability in Be star winds. A few stars have now been monitored over a baseline of nearly a decade, and there have been several opportunities for intensive observations of selected stars over periods of hours, days, or weeks.

Among the stars most thoroughly monitored for long-term variations are 59 Cygni (Doazan et al. 1985), $\gamma$ Cas (Henrichs et al. 1983; Doazan et al. 1980), 66 Oph (Barker and Marlborough 1985; Grady, Sonneborn, and Wu 1986); $\theta$ CrB (Doazan et al. 1986; Underhill 1985) and $88 \mathrm{Her}$ (Barylak and Doazan 1986). Typically what is seen on short timescales is episodic behavior; periods when little or no variation is observed (and usually no shifted discrete absorption components are present), interspersed with periods when changes occur in days or even hours, usually in the form of shifting or varying discrete absorption components. On the longer timescales, these episodes form patterns that are not strictly periodic, but may represent cyclical behavior.

The short-term variability in the discrete absorption components can take several forms: the components may appear and disappear, they may become multiple (as many as five have been seen simultaneously in one star), or they may shift in velocity, sometimes appearing near the rest velocity, and other times being displaced by several hundred $\mathrm{km} \mathrm{s}^{-1}$. 
Henrichs et al. (1983; also described by Henrichs 1984) followed an episode of shifting discrete components in $\gamma$ Cas, and were able to interprete it in terms of blobs or puffs of enhanced density flowing outward through the wind. It appeared that a blob or puff was ejected by the star and then was accelerated up to the terminal velocity of the wind over a period of several hours, and then remained at its terminal velocity for several days.

A different interpretation of the discrete absorption components has been offered by Barker (this conference), who has been able to reproduce the full variety of observed resonance line profiles by postulating a velocity law that has several decelerations due to the presence of propagating shocks, through which density enhancements (puffs) flow with the outward wind. No basis for creating the shocks is offered, but the empirical agreement between theoretical and observed profiles is striking.

The long-term variability that is observed in the ultraviolet wind indicators in Be stars is difficult to interpret. A given star may undergo periods of intense activity in the discrete absorption components, followed by lengthy intervals when nothing happens, when there may be no indication of the presence of a wind or of superionization. Probably the best chance of understanding the significance of these variations lies in comparing what happens in ultraviolet wavelengths with what is observed in the visible and infrared portions of the spectrum. Unfortunately, such comparisons have been very difficult to make, primarily because of the difficulties of scheduling different telescopes (on the ground and in space) for observations of the same target at the same time. Nevertheless, some interesting and significant results have been obtained.

In 59 Cyg (Doazan et al. 1985) and 66 Oph (Polidan and Peters 1986) variability in ultraviolet wind indicators observed with the IUE has clearly been linked with variability observed in other wavelength bands. In $59 \mathrm{Cyg}$, episodes of variability in CIV were linked with variations in the V/R ratio and the equivalent width of the $\mathrm{H} \alpha$ emission. In $66 \mathrm{Oph}$ there was an increase in CIV absorption equivalent width at the same time that the far-ultraviolet flux (below $912 \AA$ ) decreased, as observed by the Voyager 1 and 2 spacecraft. Other studies show that there is a relationship between $\mathrm{H} \alpha$ behavior and the appearance of the far-ultraviolet wind diagnostics $(\theta \mathrm{CrB}$, studied by Doazan et al. 1986; 88 Her, Barylak and Doazan 1986).

These studies show that, despite the fact that the principal wind indicators in the ultraviolet and optical wavelengths are formed in physically different regions, the cause of the variations in each region must be the same or at least closely related. It will prove especially important to pinpoint the exact sequence of perturbations that affect the different wavelength regimes (such as the ultraviolet, the optical, and the infrared), because doing so may isolate the geometric structure of the circumstellar material. This could, for example, distinguish unequivocally between the disk and spherically symmetric timedependent models described earlier. The fact that the different regions are indeed connected together by their long-term variability is perhaps the most important new development since the last Be star symposium.

\section{THE OB STAR CONNECTION}

Throughout this review, comparisons have been made between the behavior of high-energy phenomena in Be stars and similar phenomena in the more luminous OB 
stars. While there are some significant differences, the similarities are striking, and may eventually prove useful in determining the cause of the Be phenomenon.

A major similarity between $\mathrm{OB}$ and Be stars is that both groups have highvelocity stellar winds. The winds in both groups are characterized by superionization and by discrete absorption components, and in both groups these components may be ubiquitous. The mass-loss rates for the Be stars are lower, but so are the stellar luminosities; the ratio of mass-loss rate to luminosity is similar for the two groups. This suggests that radiation pressure may play a role in determining the mass-loss rate for Be stars, since it is widely believed that radiation pressure drives the winds in $O B$ stars. On the other hand, the relationship is not precisely the same, and there is considerable scatter in the correlation of mass-loss rate with luminosity for the Be stars.

One reason for the scatter in Be star correlation between mass-loss rate and luminosity is the variability of the mass loss, which apparently is due entirely to variations in the discrete absorption components. The discrete components in OB stars may be as variable as those in Be stars, but they represent a much smaller fraction of the total massloss rates for the $\mathrm{OB}$ stars.

While the similarities in mass-loss characteristics between $O B$ and Be stars suggest that both are influenced by radiation pressure, the differences make it likely that some other factor affects the Be stars. This is consistent with theoretical work showing that there is a minimum luminosity for radiatively initiated winds (Abbott 1979); Be stars fall below the theoretical cut-off. A significant new result, from the large-scale surveys cited in this review, is that the unknown factor that produces winds in Be stars does not work in normal B stars. The close link between winds and the Be phenomenon is a powerful argument that the winds and the Be characteristics have a common origin.

The Einstein Observatory data on OB stars showed them to be soft x-ray emitters, with a normal ratio of x-ray to optical luminosity of about $10^{-7}$. (Vaiana et al. 1981). Because of the similarities of $\mathrm{OB}$ and $\mathrm{Be}$ winds and superionization, and suggestions that the superionization in OB stars is due to ionization by soft $x$-rays (produced either in a corona or by shocks in the wind), the question arises as to whether Be stars are also soft $\mathrm{x}$ ray sources. Unfortunately, $x$-ray data available now are not sensitive enough to answer this question; a ratio of $\mathrm{x}$-ray to optical luminosity of $10^{-7}$ for Be stars places them generally below the detection threshold for Einstein and EXOSAT. A search for x-ray emission from Be stars should be attempted with future instruments such as ROSAT and $A X A F$.

\section{SUMMARY AND RECOMMENDATIONS FOR FUTURE WORK}

Out of all the data from surveys of numbers of Be stars, and from the complex detailed behavior of a few well-studied individuals, come a few general conclusions that today seem safe to make:

(1) High-energy phenomena in Be stars are associated with the Be phenomenon itself. Among $B$ stars, Be stars have stronger winds and a greater degree of ionization than the normal $\mathrm{B}$ stars. Discrete aborption components occur exclusively in $\mathrm{Be}$ stars.

(2) High-energy phenomena are either latitude-dependent or initiated by rotation, or both. Significant relationships between the presence of winds and discrete absorption components and vsini demonstrate this. 
(3) High-energy phenomena in Be stars have strong similarities with highenergy phenomena in the more luminous $O B$ stars. This suggests that radiation pressure may play a role in driving the Be star winds, but contrasts between $O B$ and $B e$ stars indicate that some other factor must be involved in the Be stars.

Probably the most important recommendation for future studies is that intensive multi-spectral monitoring of selected Be stars be carried out on many timescales. Comparisons of data from the ultraviolet, optical, infrared, and possibly the $\mathrm{x}$-ray portions of the spectrum have the potential for distinguishing between competing geometrical models of the circumstellar material in Be stars, and possibly for isolating the cause of the Be phenomenon itself. Because the flow time for material to accelerate through the wind to a height of several stellar radii is of order a few days, it may eventually prove feasible to make continuous multi-spectral observations of a star long enough to observe a transient event, such as the formation of a discrete absorption component, as it transits the circumstellar material to such a height. This might answer many of the questions about the relationship between the high-energy phenomena, observed in the ultraviolet and the other Be signatures observed in the optical and the infrared. On the other hand, repeated observations over times of years or decades may tell us the true nature of the episodic behavior of Be stars, the nature of the changes that convert a normal B star into a Be or shell star.

Looking ahead to a time when new astronomical instruments may be designed for space operations, a multi-spectral mission consisting of optical, ultraviolet, infrared, and x-ray telescopes, all coaligned, would be a marvelous tool for exploring the nature of Be stars. Such a mission might be readily accomodated by the free-flying coorbital platforms being studied as part of the U.S. space station program, so we may find an opportunity to develop such a mission within a decade or so. Until then, we must keep making the best use possible of existing instruments, which for now (as long as IUE keeps operating) allows us access to the optical, the near infrared, and the ultraviolet. There will, no doubt, be many new discoveries from these wavelength bands to be reported at the next conference on Be stars.

\section{REFERENCES}

Abbott, D. C. 1979, Mass Loss and' Evolution in O-Type Stars, IAU Symposium 89, eds. P.S. Conti and C.W.H. de Loore (Dordrecht: Reide1), p. 237.

Barker, P. K. and Marlborough, J. M. 1985, Ap.J., 288, 329.

Barker, P. K., Marlborough, J. M., and Landstreet, J. D. 1984, The Future of Ultraviolet Astronomy Based on Six Years of IUE Research, NASA CP-2949, eds. J.L. Mead, R.D. Chapman, and Y. Kondo (Washington:NASA), p. 219.

Barylak, M. and Doazan, V. 1986, Astr. Ap., in press.

Cassinelli, J. P. 1979, Ann. Rev. Astr. Ap., 17, 275.

Cassinelli, J. P. and Olson, G. L. 1979, Ap.J., 229, 304.

Cassinelli, J. P., Olson, G. L., and Stalio, R. 1978, Ap.J., 220, 573.

Castor, J. I. and Lamers, H. J. G. L. M. 1979, Ap.J. Supple., 39, 481. 
Doazan, V., Grady, C. A., Snow, T. P., Peters, G. J., Marlborough, J. M., Barker, P. K., Bolton, C. T., Bourdonneau, B., Kuhi, L. V., Lyons, R. W., Polidan, R. S., Stalio, R., and Thomas, R. N. 1985, Astr. Ap., 152, 182.

Doazan, V., Marlborough, J. M., Morossi, C., Peters, G. J., Rusaconi, L., Sedmak, G., Stalio, R., Thomas, R. N., and Willis, A. 1986, Astr. Ap., 158, 1.

Grady, C. A., Bjorkman, K. S., and Snow, T. P. 1987, Ap.J., submitted.

Grady, C. A., Sonneborn, G., and Wu, C.-C. 1986, preprint.

Henrichs, H. F. 1984, Proc. Fourth European IUE Conference, ESA SP-218, eds. E. Rolfe and E. Battrick (Rome:ESA), p. 43.

Henrichs, H. F. 1986, O, Of, and Wolf-Rayet Stars, NASA/CNRS Monograph Series, eds. P.S. Conti and A.B. Underhill (Washington:NASA), in press.

Henrichs, H. F., Hammerschlage-Hensberge, G., Howarth, I. D., and Barr, P. 1983, Ap.J., 268, 807.

Hubeny, I., Harmanec, P., and Stefl, S. 1986, preprint.

Jaschek, M and Groth, H.-G. (eds.), 1982, Be Stars, IAU Symposium 98 (Dordrecht:Reidel).

Lamers, H. J. G. L. M., Gathier, R., and Snow, T. P. 1982, Ap.J., 258, 186.

Lamers, H. J. G. L. M. and Snow, T. P. 1978, Ap.J., 219, 504.

Lucy, L. B. 1982, Ap.J., 255, 286.

Lucy, L. B. and White, R. L. 1980, Ap.J., 241, 300.

Marlborough, J. M. 1977, Ap.J., 216, 446.

Marlborough, J. M. 1982, Be Stars, IAU Symposium 98, eds. M. Jaschek and H.-G. Groth (Dordrecht: Reide1), p. 361 .

Marlborough, J. M. and Peters, G. J. 1982, Be Stars, IA U Symposium 98, eds. M. Jaschek. and H.-G. Groth (Dordrecht: Reide1), p. 387.

Marlborough, J. M. and Snow, T. P. 1980, Ap.J., 235, 85.

Morton, D. C. 1976, Ap.J., 203, 386.

Polidan, R. S. and Peters, G. J. 1986, private communication.

Prinja, R. K. and Howarth, I. D. 1986, Astr. Ap. Suppl., in press.

Rogerson, J. B. and Lamers, H. J. G. L. M. 1975, Nature, 256, 190.

Slettebak, A. and Carpenter, K. G. 1983, Ap.J. Suppl., 53, 869.

Slettebak, A. and Snow, T. P. 1978, Ap.J. (Lett.), 224, L127.

Snow, T. P. 1981, Ap.J., 251, 139.

Snow, T. P. 1982, Ap.J. (Lett.), 253, L39.

Snow, T. P. and Marlborough, J. M. 1976, A p.J. (Lett.), 203, L87.

Snow, T. P. and Morton, D. C. 1976, Ap.J. Suppl., 32, 429. 
Snow, T. P. and Stalio, R. 1987, Scientific Accomplishments of the IUE, eds. Y. Kondo, A. Boggess, C. de Jager, M. Grewing, A.L. Lane, J.L. Linsky, W. Wamsteker, and $\mathrm{R}$. Wilson, in press.

Underhill, A.B. 1985, Astr. Ap., 148, 431.

Vaiana, G. S., Cassinelli, J. P., Fabbiano, G., Giacconi, R., Golub, L., Gorenstein, P., Haisch, B. M., Harnden, F. R., Johnson, H. M., Linsky, J. L., Maxson, C. W., Mewe, R., Rosner, R., Seward, F., Topka, K., and Zwaan, C. 1981, Ap.J., 245, 163. 


\section{DISCUSSION FOLLOWING SNOW}

\section{Harmanec:}

I am still convinced that you cannot use the UV resonance lines for the determination of the mass loss rates because of heavy line blending. Modeling by Hubeny et al. (1985, 1986) clearly shows that line blending is responsible for violet- extended wings of both SiIV lines for vsini of about $100 \mathrm{kms}^{-1}$, and for red-extended rings for vsini $\sim 300 \mathrm{kms}^{-1}$. As to the CIV lines, we found a disagreement of model and observed spectra always for the $1548 \AA$ line. It is notable that the data on $\theta \mathrm{CrB}$ by Doazan et al. (1986) indeed show that at some epochs there was more absorption at $1548 \AA$ but no absorption corresponding to the other CIV line. This may indicate some shell unidentified feature exists there. Also, the correlation of the CIV and the $\mathrm{H} \alpha$ strength for $59 \mathrm{Cyg}$ may possibly indicate that the variations of the cool envelope are responsible for the variations seen near $1550 \AA$.

Snow:

I am willing to admit that in some cases line blends confuse the interpretation of UV resonance lines, but I am not willing to concede that this is generally a problem. I don't see how blends can simulate all the observed asymmetries, and it is especially difficult to see how blends can simulate the variation of discrete absorption components associated with UV resonance lines.

\section{Doazan:}

I would like to comment on the "spurious" CIV resonance lines attributed to blends of FeIII and FeII by Harmanec et al. It is easy to check the reality of the CIV lines on IUE spectra by inspecting the FeIII lines of multiplet 34 and FeII resonance lines. In order to obtain a CIV "spurious" feature of the intensity these authors predict from their synthetic spectra, I would think that the FeIII line of multiplet 34 and the FeII resonance line would be almost saturated, which is not the case in several stars. These authors do not show what strength they predict for these strong lines, as is usually done in spectroscopic work. Therefore, I would be very suspicious when considering their results.

Grady:

UV observations of variability in the vicinity of CIV, SiIV, NV, and SiIII are common. Line profile variations outside the resonance profiles are not seen to within the $\mathrm{S} / \mathrm{N}$ limits of the IUE. These data tend to suggest that line blending by variable FeII and FeIII is not present, since these species have many lines outside the vicinity of the UV resonance lines.

Snow:

Right: does anyone else want to help defend me?

Bolton:

I remember frequent comments on the line blending problem at the Munich meeting, so the problem has been well known for a long time. However, many, if not most, of the papers on superionized lines do not mention any steps to control this problem. Earlier remarks in this discussion indicate that these steps have been taken in many cases, but since these are not documented in the papers, it is impossible for those of us who are not intimately familiar with the data to judge the validity of the conclusions that are drawn. It would help if authors would describe any steps taken to determine the effect of line blending.

Snow:

I agree. 


\section{Underhill:}

You mentioned ways of generating discrete components. May I add the proposal by me and Fahey (1984) that the discrete components originate from the release of plasma from points on or above the surface of a rotating star? We inferred the presence of locally distributed magnetic fields (BMR's) in the stellar surface. The presence of such magnetic fields is an asset for providing the hot plasma seen by means of superionization, $x$-rays and so on.

Snow:

I agree that magnetic structures potentially may play an important role in producing discrete absorption components.

Henrichs:

I want to add a few remarks to your talk. (1) It cannot be emphasized enough that all the CIV absorption excess you mentioned is actually in the form of variable discrete absorption components. (2) Variable CIV absorption among B (non-supergiant) stars is only encountered in Be stars. This is in my opinion a very fundamental point. (3) You did not mention the timescales of variability. The typical shortest timescale is of the order of less than a half a day, i.e. comparable to the flow timescale of the material. (4) You mentioned only a very few models for explaining the discrete components. There are many more, all of them equally well capable of explaining some of the observed properties. (5) There are at least three documented cases of correlated behavior of the discrete components, $\mathrm{H}$ behavior and changes in nonradial pulsation properties.

Snow:

Yes, I agree. I think the biggest news is that we can now say with certainty that the superionization and discrete components are definitely linked to the Be phenomenon. This was not yet clear five years ago, at the last conference. Incidentally, I am not convinced that the CIV absorption in all cases consists entirely of discrete components; I think that in many cases there is a broad, stable feature also present.

Bruhweiler:

You might add that the phenomenon of discrete, displaced components, as evidence of mass loss, is also seen in O subdwarfs such as HD 128220B (see Bruhweiler and Dean 1983). It is also seen in hot white dwarfs, except at much lower velocities (Bruhweiler and Kondo 1983)).

Snow:

Thank you. 